\title{
A Continuous-Time Model for Reinvestment Risk in Bond Markets
}

\author{
Mikkel Dahl \\ Laboratory of Actuarial Mathematics, University of Copenhagen, \\ Universitetsparken 5, DK-2100 Copenhagen Ø, Denmark. \\ Email:dahl@math.ku.dk
}

1st August 2005

\begin{abstract}
We propose a bond market model, where, as in practice, only bonds with a limited time to maturity are traded in the market. As time passes, new bonds with stochastic initial prices are introduced in the market. Hence, we are able to model the reinvestment risk present in practice, when considering long term contracts. To quantify and control the reinvestment risk we apply the criterion of risk-minimization.
\end{abstract}

JEL classification: G10.

Mathematics Subject Classification (2000): 62P05, 91B28.

Key words: Forward rates, Heath-Jarrow-Morton framework, incomplete market, riskminimization.

\section{Introduction}

In the literature, bond markets are usually assumed to include all bonds with time of maturity less than or equal to the time of maturity of the considered claim. However, in practice only bonds with a limited (sufficiently short) time to maturity are traded. Hence, standard models are only adequate to describe pricing and hedging of so-called short term contracts, where the payoff depends on bonds with time to maturity less than or equal to the longest traded bond. When considering long term contracts, where the payoff depends on bonds with longer time to maturity than the longest traded bond, the bond market does not in general include bonds which at all times allow for a perfect hedge of the contract. Thus, in practice, an agent interested in pricing and hedging 
long term contracts is exposed to a reinvestment risk, which is ignored in standard bond market models. Here, the reinvestment risk refers to the uncertainty associated with the obtainable rate of return, when reinvesting in bonds not yet traded in the market. An example of long term contracts sold in practice are life insurance contracts, where the liabilities of the insurance companies often extend 50 years, or more, into the future. In this paper, we propose a model, where pricing and hedging of short term contracts is similar to a standard bond market model, whereas the model includes reinvestment risk, when considering long term contracts.

In order to describe the reinvestment risk, we initially consider a standard continuous-time bond market model with some fixed finite time horizon, which is less than (or equal to) the time horizon of the considered payment process. At fixed times new bonds are issued in the market, such that we immediately after the issue of new bonds consider a standard model identical to the initial one. The entry prices of the new bonds depend on the prices of the bonds already traded and a stochastic term. As is standard in bond market literature, we model the forward rates rather than the bond prices themselves. Between the times of issue, the forward rates follow a standard Heath-Jarrow-Morton model, see Heath, Jarrow and Morton (1992). When new bonds are issued, the forward rate curve is extended. We assume that at each time of issue the extension is continuous and depends on a single random variable. The idea of fixing the maximum time to maturity of the traded assets and introducing new assets as times passes can also be found in Neuberger (1999), who considers a market for futures on oil prices. Neuberger models the initial price of the new future as a linear function of prices on traded futures and a normally distributed error term.

To the author's knowledge the only other papers considering the problem of modelling the prices of newly issued bonds are Sommer (1997) and Dahl (2005). Dahl (2005) considers a discrete-time model for the reinvestment risk, whereas Sommer (1997) considers a continuous-time bond market. A major difference between Sommer (1997) and this paper is the way new bonds are issued and priced in the market. While Sommer (1997) considers the case where new bonds are issued continuously, this paper, as is the case in practice, considers a set of fixed times, where new bonds are issued. Hence, the present model should be more apt to describe practice. Within his setup Sommer derives conditions on the forward rate dynamics in order to have sufficiently smooth forward rate curves and risk-minimizing strategies.

To quantify and control the reinvestment risk associated with long term contracts, we apply the criterion of risk-minimization introduced by Föllmer and Sondermann (1986) for contingent claims and extended in Møller (2001b) to the case of payment processes. The derivation of the risk-minimizing strategies are based on the ideas of Schweizer (1994) regarding risk-minimization under restricted information. Hence, the risk-minimizing strategies are given in terms of the replicating strategies in the case without reinvestment risk.

The paper is organized as follows: In Section 2 a bond market model including reinvestment risk is introduced. This is done in two steps: First we describe a standard bond market model, and then the model is extended to include reinvestment risk. In this section we also introduce the considered class of equivalent martingale measures and the relevant financial terminology. Risk-minimizing strategies are derived in Section 3, and we conclude the paper by describing a possible implementation of the model in Section 4 . 


\section{The bond market model}

Let $\widehat{T}$ be a fixed finite time horizon and $(\Omega, \mathcal{F}, P)$ a probability space with a filtration $\mathbb{F}=\left(\mathcal{F}_{t}\right)_{0 \leq t \leq \widehat{T}}$ satisfying the usual conditions of right-continuity, i.e. $\mathcal{F}_{t}=\bigcap_{u>t} \mathcal{F}_{u}$, and completeness, i.e. $\mathcal{F}_{0}$ contains all $P$-null sets.

\section{$2.1 \quad$ A standard model}

Consider another fixed time horizon $\widetilde{T}, \widetilde{T} \leq \widehat{T}$, and a bond market, where at time $t$, $0 \leq t \leq \widetilde{T}$ all zero coupon bonds with maturity $\tau, t \leq \tau \leq \widetilde{T}$ are traded. Let $P(t, \tau)$ denote the price at time $t$ of a zero coupon bond maturing at time $\tau$. To avoid arbitrage we assume that $P(t, \tau)$ is strictly positive and $P(t, t)=1$ for all $t$. For non-negative interest rates the price $P(t, \tau)$ is a decreasing function of $\tau$ for fixed $t$. An important quantity when modelling bond prices is the (instantaneous) forward rate with maturity $\tau$ contracted at time $t$ defined by

$$
f(t, \tau)=-\frac{\partial \log P(t, \tau)}{\partial \tau}
$$

or, stated differently,

$$
P(t, \tau)=e^{-\int_{t}^{\tau} f(t, u) d u}
$$

The forward rate $f(t, \tau)$ can be interpreted as the riskfree interest rate, contracted at time $t$ over the infinitesimal interval $[\tau, \tau+d \tau)$. The short rate process $\left(r_{t}\right)_{0<t<\widetilde{T}}$ is defined as $r_{t}=f(t, t)$. Since it is inconvenient to model the dynamics of bond prices directly, the common approach in the literature is to model interest rates. Here, we take the approach of Heath et al. (1992) where the dynamics of not only the short rate but the entire forward rate curve are modelled. The connection between the forward rates and bond prices established in (2.1) and (2.2) then gives the dynamics of the bond prices. For fixed $\tau, 0 \leq t \leq \tau \leq \widetilde{T}$, the $P$-dynamics of the forward rates are given by

$$
d f(t, \tau)=\alpha^{P}(t, \tau) d t+\sigma(t, \tau) d W_{t}^{P},
$$

where $W^{P}$ is a Wiener process under $P$. For simplicity $W^{P}$ is assumed to be 1-dimensional. The processes $\alpha^{P}$ and $\sigma$ are adapted to the filtration $\mathbb{G}=\left(\mathcal{G}_{t}\right)_{0 \leq t \leq \widetilde{T}}$, which is the $P$ augmentation of the natural filtration generated by the Wiener process, i.e. $\mathcal{G}_{t}=\mathcal{G}_{t}^{+} \vee \mathcal{N}$, where $\mathcal{N}$ is the $\sigma$-algebra generated by all $P$-null sets and

$$
\mathcal{G}_{t}^{+}=\sigma\left\{W_{u}^{P}, u \leq t\right\} .
$$

Using Björk (2004, Proposition 20.5) we obtain the following $P$-dynamics for the price process of a bond with maturity $\tau$ :

$$
\begin{aligned}
d P(t, \tau)= & \left(r_{t}-\int_{t}^{\tau} \alpha^{P}(t, u) d u+\frac{1}{2}\left(\int_{t}^{\tau} \sigma(t, u) d u\right)^{2}\right) P(t, \tau) d t \\
& -\int_{t}^{\tau} \sigma(t, u) d u P(t, \tau) d W_{t}^{P}, \quad 0 \leq t \leq \tau \leq \widetilde{T}
\end{aligned}
$$


In addition to the bonds, we assume that the financial market includes a savings account earning the short rate $r$. The dynamics of the savings account are

$$
d B_{t}=r_{t} B_{t} d t, \quad B_{0}=1 .
$$

Remark 2.1 The existence of a savings account with drift $r$ can be proven if we allow for investments in infinitely many different bonds. In this case, investing in a roll-over strategy in just-maturing bonds produces a value process, whose dynamics are given by (2.5), see Björk, Kabanov and Runggaldier (1997).

For any $\mathbb{G}$-adapted process $h$ we may define a likelihood process $\left(\Lambda_{t}\right)_{0 \leq t \leq \widetilde{T}}$ by

$$
\Lambda_{t}=e^{-\frac{1}{2} \int_{0}^{t} h_{u}^{2} d u+\int_{0}^{t} h_{u} d W_{u}^{P}} .
$$

It is well known, see e.g. Musiela and Rutkowski (1997) and Björk (2004), that if there exists an $h$ such that $E^{P}\left[\Lambda_{\widetilde{T}}\right]=1$ and, for all $0 \leq t \leq \tau \leq \widetilde{T}$, the Heath-Jarrow-Morton (HJM) drift condition

$$
\alpha^{P}(t, \tau)=\sigma(t, \tau)\left(\int_{t}^{\tau} \sigma(t, u) d u-h_{t}\right)
$$

holds, then there exists a unique equivalent martingale measure $Q$ given by

$$
\frac{d Q}{d P}=\Lambda_{\widetilde{T}}
$$

Here, it is important that for fixed $t,(2.6)$ holds simultaneously for all $\tau, 0 \leq t \leq \tau \leq$ $\widetilde{T}$. Recall that an equivalent martingale measure fulfills three requirements: Firstly, it is equivalent to $P$. Secondly, all discounted price processes are martingales under the new measure and lastly, it is a probability measure. If there exists a unique equivalent martingale measure the model is arbitrage free and complete, see e.g. Björk (2004, Chapter $10)$.

\subsection{Extending the standard model to include reinvestment risk}

We now extend the standard model in Section 2.1 to include reinvestment risk. The idea is as follows: Assume that at time 0 the bond market can be described by the standard model introduced in Section 2.1. At some predetermined times new bonds are issued in the market, such that immediately after the issue the bond market is given by a standard model identical to the one at time 0 . To introduce reinvestment risk in the model the initial prices of the new bonds issued depend on a random variable independent of the observable bond prices.

In order to extend the bond market we introduce the fixed time horizon $T, \widetilde{T} \leq T \leq \widehat{T}$. The interpretation of the time horizons is as follows: $\widehat{T}$ is the last time where trading is possible in the bond market, i.e. $\widehat{T}$ may be thought of as "the end of the world", $T$ is the last time at which we allow payments and $\widetilde{T}$ is the upper limit for the time to maturity of a bond traded in the market. Hence, at any time $t$ the time to maturity of the longest traded 
bond is less than or equal to $\widetilde{T}$. Now define the sequence $0=T_{0}<T_{1}<\ldots<T_{n} \leq T$ of times, where new bonds are issued in the market. At time $T_{i}$ new bonds are issued such that all bonds with time to maturity less than or equal to $\widetilde{T}$ are traded. To ensure that at any time, bonds are traded in the market, we assume that $\widetilde{T} \geq \max _{i=1, \ldots, n}\left(T_{i}-T_{i-1}\right)$ and $\widehat{T}=T_{n}+\widetilde{T}$.

The illustration in Figure 2.1 shows one possible ordering of $T_{1}, \ldots, T_{n}, T$ and $\widetilde{T}$ in the case $n=3$.

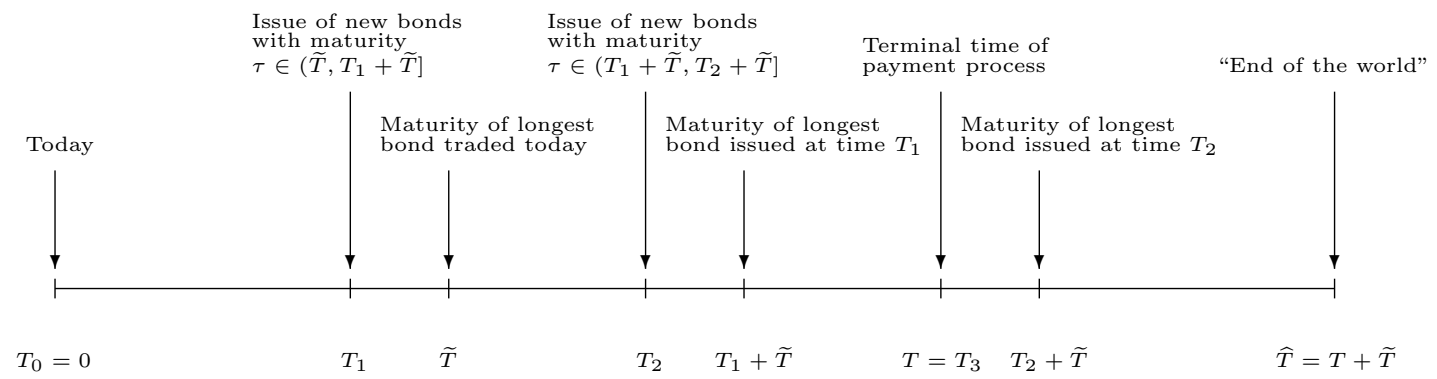

Figure 2.1: Illustration of $T_{1}, T_{2}, T_{3}, \widetilde{T}, T$ and $\widehat{T}$.

For fixed $t$ we define

$$
i_{t}=\sup \left\{0 \leq i \leq n \mid T_{i} \leq t\right\}
$$

such that $T_{i_{t}}$ is the last time new bonds are issued prior to time $t$ (time $t$ included). Thus, at time $t$ the time of maturity, $\tau$, of the bonds traded in the bond market satisfies $t \leq \tau \leq T_{i_{t}}+\widetilde{T}$. For an illustration of $T_{i_{t}}$ see Figure 2.2.

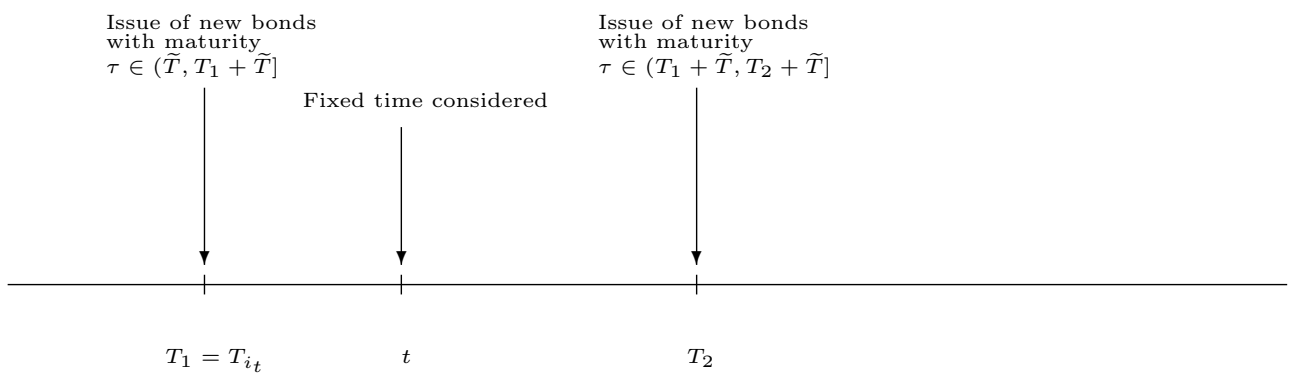

Figure 2.2: Illustration of $T_{i_{t}}$.

When the forward rates are defined, i.e. for $0 \leq t \leq \tau \leq T_{i_{t}}+\widetilde{T}$, their dynamics are given by

$$
d f(t, \tau)=\alpha^{P}(t, \tau) d t+\sigma(t, \tau) d W_{t}^{P},
$$

where the processes $\alpha^{P}$ and $\sigma$ are $\mathbb{F}$-adapted, and $W^{P}$ is a 1-dimensional Wiener process under $P$. As in Section 2.1 the filtration $\mathbb{G}=\left(\mathcal{G}_{t}\right)_{0 \leq t \leq \widehat{T}}$ is the $P$-augmentation of the natural filtration generated by the Wiener process. Note that $\mathbb{G}$ and the short rate process 
$\left(r_{t}\right)_{0 \leq t \leq \widehat{T}}$ are defined until time $\widehat{T}$. As noted above, forward rates for all maturities are not defined at time 0 . They are introduced at the times of issue of new bonds. To model the initial value of the new forward rates at time $T_{i}, i \in\{1, \ldots, n\}$, we introduce a sequence $Y=\left(Y_{i}\right)_{i=1, \ldots, n}$ of mutually independent random variables with distribution functions $\left(F_{i}^{P}\right)_{i=1, \ldots, n}$. Assume that $Y$ and $W^{P}$ are independent (as discussed below this is no restriction). Here, $Y_{i}$, which is revealed at time $T_{i}$, describes the uncertainty independent of the observed bond prices associated with the initial prices of bonds issued at time $T_{i}$. The filtration $\mathbb{H}=\left(\mathcal{H}_{t}\right)_{0 \leq t \leq \widehat{T}}$ is defined as the $P$-augmentation of the natural filtration generated by the random variables $\left(Y_{i}\right)_{i=1, \ldots, n}$, i.e. $\mathcal{H}_{t}=\mathcal{H}_{t}^{+} \vee \mathcal{N}$, where

$$
\mathcal{H}_{t}^{+}=\sigma\left\{\left(Y_{i}\right)_{i=1, \ldots, i_{t}}\right\} .
$$

We now assume that $\mathbb{F}$ is the total filtration generated by the bond market, such that

$$
\mathcal{F}_{t}=\mathcal{G}_{t} \vee \mathcal{H}_{t}
$$

For $T_{i-1}+\widetilde{T}<\tau \leq T_{i}+\widetilde{T}$ we model the forward rates by

$$
f\left(T_{i}, \tau\right)=f\left(T_{i}, T_{i-1}+\widetilde{T}\right)+\int_{T_{i-1}+\widetilde{T}}^{\tau} \gamma_{u}^{i} d u
$$

where $\gamma^{i}$ is an $\mathcal{F}_{T_{i}}$-measurable function, i.e. each $\gamma_{u}^{i}$ is $\mathcal{F}_{T_{i}}$-measurable for $u \in\left(T_{i-1}+\widetilde{T}, T_{i}+\right.$ $\widetilde{T}]$. The interpretation is that the new forward rates introduced at time $T_{i}$ depend on the past forward rates and some noise represented by the random variable $Y_{i}$. The assumed independence between $W^{P}$ and $Y$ is no restriction, since we otherwise could define a vector of random variables $\widetilde{Y}=\left(\widetilde{Y}_{i}\right)_{i=1, \ldots, n}$ independent of $W^{P}$ and functions $\widetilde{\gamma}^{1}, \ldots, \widetilde{\gamma}^{n}$, such that $\widetilde{f}\left(T_{i}, \tau\right)$ given by $(2.8)$ with $\gamma$ and $Y$ replaced by $\widetilde{\gamma}$ and $\tilde{Y}$, respectively, has the same distribution as $f\left(T_{i}, \tau\right)$ for $T_{i-1}+\widetilde{T}<\tau \leq T_{i}+\widetilde{T}$. We note from (2.8) that the forward rate curve is continuous at all times. In addition to $\mathbb{F}$ we consider the filtrations $\mathbb{F}^{T_{i}}$, $i \in\{0,1, \ldots, n\}$, given by

$$
\mathbb{F}^{T_{i}}=\left(\mathcal{F}_{t}^{T_{i}}\right)_{0 \leq t \leq \widehat{T}}=\left(\mathcal{H}_{T_{i}} \vee \mathcal{F}_{t}\right)_{0 \leq t \leq \widehat{T}}
$$

We immediately note that $\mathbb{F}=\mathbb{F}^{T_{0}}=\mathbb{F}^{0}$. For $i \geq 1$ the interpretation of the filtration $\mathbb{F}^{T_{i}}$ is that the sequence $\left(Y_{j}\right)_{j=1, \ldots, i}$ is known at time 0 . When considering $\mathbb{F}^{T_{n}}$ the entire vector $Y$ is known at time 0 , so the model is complete. Furthermore, we note that if we for $i \in\{0, \ldots, n-1\}$ consider the filtration $\mathbb{F}^{T_{i}}$ and the time interval $\left[0, T_{i+1}\right)$, then the model is complete.

In the extended bond market we have that for any $i$ the outcome of the random variable $Y_{i}$ affects the initial prices of bonds issued at time $T_{i}$, and once it is realized it may affect the drift and the volatility of the forward rates. Thus, prior to time $T_{i}$, where $Y_{i}$ is realized, we are unable to trade in assets depending on the outcome of $Y_{i}$. Hence, the vector $Y$ is unhedgeable. Once the forward rates are introduced, the dynamics of the bonds are driven solely by $W^{P}$. Thus, the model can be viewed as a series of complete models on $\left[T_{i}, T_{i+1}\right)$ and a vector of independent random variables realized at times $T_{i}, i \in\{1, \ldots, n\}$.

Remark 2.2 As noted above the model is complete when considering $\mathbb{F}^{T_{n}}$. Hence, contingent on the outcome of $Y$, all zero coupon bonds have unique prices at all times (even before they are traded). Thus, at time $t, 0 \leq t<T_{n}$, where the unconditional model is incomplete, we have a forward rate curve for all maturities in the conditional model. Here, 
we note that all conditional forward rate curves, of which there may be infinitely many, are identical until time $T_{i_{t}}+\widetilde{T}$. However, in the unconditional model, the future values of $Y_{i_{t}+1}, \ldots, Y_{n}$ are unknown. Hence, it is uncertain which of the conditional forward rate curves will turn out in retrospect to have been "the correct one" when $Y_{i_{t}+1}, \ldots, Y_{n}$ have been observed at time $T$. Thus, we can interpret the reinvestment risk as the uncertainty associated with which of the conditional forward rate curves in retrospect has turned out to have been "the correct one". This in turn gives that the magnitude of the reinvestment risk is related to how much the conditional forward rate curves differ.

We now derive an expression for future forward rates, and in particular future short rates, in terms of the present forward rates and the future uncertainty. For fixed $\tau$ we define

$$
\bar{i}_{\tau}=\inf \left\{0 \leq i \leq n \mid T_{i}+\widetilde{T} \geq \tau\right\},
$$

such that $T_{\bar{i}_{\tau}}$ is the first time a bond with maturity $\tau$ is traded. Hence, the initial time a forward rate with maturity $\tau$ is defined. For $0 \leq t \leq u \leq \tau \leq T_{i_{t}}+\widetilde{T}$ we have the well-known relation

$$
f(u, \tau)=f(t, \tau)+\int_{t}^{u} d f(s, \tau) .
$$

However, as can be seen from the following proposition, the relationship between the forward rates is in general more involved, since the future forward rates depend on the entry prices of bonds yet to be issued. In the proposition, and throughout the paper, we interpret $\sum_{k=j}^{\ell}$ as 0 if $\ell<j$.

\section{Proposition 2.3}

For $0 \leq t \leq u \leq \tau$ we have the following relation between the forward rates:

$$
\begin{aligned}
f(u, \tau)= & f\left(t,\left(T_{i_{t}}+\widetilde{T}\right) \wedge \tau\right)+\sum_{k=i_{t}}^{\bar{i}_{\tau}-1}\left(\int_{t \vee T_{k}}^{T_{k+1}} d f\left(s, T_{k}+\widetilde{T}\right)+\int_{T_{k}+\widetilde{T}}^{\tau \wedge\left(T_{k+1}+\widetilde{T}\right)} \gamma_{s}^{k+1} d s\right) \\
& +\int_{T_{\bar{i}_{\tau}} \vee t}^{u} d f(s, \tau) .
\end{aligned}
$$

In particular it holds for the short rate that

$$
\begin{aligned}
r_{u}= & f\left(t,\left(T_{i_{t}}+\widetilde{T}\right) \wedge u\right)+\sum_{k=i_{t}}^{\bar{i}_{u}-1}\left(\int_{t \vee T_{k}}^{T_{k+1}} d f\left(s, T_{k}+\widetilde{T}\right)+\int_{T_{k}+\widetilde{T}}^{u \wedge\left(T_{k+1}+\widetilde{T}\right)} \gamma_{s}^{k+1} d s\right) \\
& +\int_{T_{\bar{i}_{u} \vee t}}^{u} d f(s, u) .
\end{aligned}
$$

Proof of Proposition 2.3: Formula (2.10) follows by repeated use of relations (2.8) and (2.9), whereas the expression for the short rate in (2.11) is obtained by setting $\tau=u$ in (2.10). 


\subsubsection{A class of equivalent martingale measures}

In this section we introduce the considered class of equivalent martingale measures. First we determine the unique Girsanov kernel with respect to the Wiener process and define the equivalent martingale measure corresponding to a change of measure with respect to the Wiener process only. We then consider a change of measure with respect to $Y$ as well. Since $Y$ is unhedgeable there exist infinitely many equivalent martingale measures. Here, we consider a class of measures with particular nice properties.

Similarly to the standard model we observe that the existence of an equivalent martingale measure depends on the existence of an $\mathbb{F}$-adapted process $h$, such that for all $0 \leq t \leq \tau \leq$ $T_{i_{t}}+\widetilde{T}$ the HJM drift condition

$$
\alpha^{P}(t, \tau)=\sigma(t, \tau)\left(\int_{t}^{\tau} \sigma(t, u) d u-h_{t}\right)
$$

is satisfied, and the likelihood process $\Lambda=\left(\Lambda_{t}\right)_{0 \leq t \leq \widehat{T}}$ defined by

$$
\Lambda_{t}=e^{-\frac{1}{2} \int_{0}^{t} h_{u}^{2} d u+\int_{0}^{t} h_{u} d W_{u}^{P}}
$$

fulfills $E^{P}\left[\Lambda_{\widehat{T}}\right]=1$. Hence, if such an $h$ exists, we may define an equivalent martingale measure $Q^{0}$ by

$$
\frac{d Q^{0}}{d P}=\Lambda_{\widehat{T}}
$$

However, the equivalent martingale measure $Q^{0}$ is not unique. In particular we can define another likelihood process $U=\left(U_{t}\right)_{0 \leq t \leq \widehat{T}}$ by

$$
U_{t}=\prod_{j=1}^{i_{t}}\left(1+u_{j}\left(Y_{j}\right)\right),
$$

for some functions $u_{j}, j \in\{1, \ldots, n\}$, satisfying $u_{j}(y)>-1$ for all $y$ in the support of $Y_{j}$ and $E^{P}\left[u_{j}\left(Y_{j}\right)\right]=0$. Here, and henceforth, $\prod_{j=k}^{\ell}$ is interpreted as 1 if $k>\ell$. If $E^{Q^{0}}\left[U_{\widehat{T}}\right]=1$ (or equivalently $E^{P}\left[\Lambda_{\widehat{T}} U_{\widehat{T}}\right]=1$ ), we can define an equivalent martingale measure $Q$ by

$$
\frac{d Q}{d Q^{0}}=U_{\widehat{T}}
$$

Girsanov's theorem gives that for any $Q$ of the form (2.13), the process

$$
W_{t}^{Q}=W_{t}^{P}-\int_{0}^{t} h_{u} d u
$$

is a Wiener process. Moreover, the distribution function of $Y_{i}, i \in\{1, \ldots, n\}$, under $Q$, $F_{i}^{Q}$, is given by

$$
F_{i}^{Q}(y)=\int_{-\infty}^{y}\left(1+u_{i}(z)\right) d F_{i}^{P}(z)
$$

Here, we restrict ourselves to the case, where $h$ is $\mathbb{G}$-adapted, such that the measures considered are particularly simple, since $Y$ and $W^{Q}$ are independent under $Q$ and the 
mutual independence of the $Y_{i}$ 's is preserved under $Q$. Using (2.14) we find that the dynamics of the forward rates under $Q$ are given by

$$
d f(t, \tau)=\alpha^{Q}(t, \tau) d t+\sigma(t, \tau) d W_{t}^{Q}
$$

where we have defined

$$
\alpha^{Q}(t, \tau)=\sigma(t, \tau) \int_{t}^{\tau} \sigma(t, u) d u
$$

Now, Björk (2004, Proposition 20.5) gives the following bond price dynamics for $0 \leq t \leq$ $\tau \leq T_{i_{t}}+\widetilde{T}$ under $Q$ :

$$
d P(t, \tau)=r_{t} P(t, \tau) d t-\int_{t}^{\tau} \sigma(t, u) d u P(t, \tau) d W_{t}^{Q} .
$$

Remark 2.4 It can be shown that $Q^{0}$ defined by (2.12) is the so-called minimal martingale measure for the extended model, i.e. the equivalent martingale measure which "disturbs the structure of the model as little as possible", see Schweizer (1995).

\subsection{Model considerations}

In this section we comment on the model specification in Section 2.2. At any time $t$ the prices of bonds with maturity $\tau, t<\tau \leq T_{i_{t}}+\widetilde{T}$, must satisfy both

$$
P(t, \tau)=e^{-\int_{t}^{\tau} f(t, u) d u}
$$

and

$$
P(t, \tau)=E^{Q}\left[e^{-\int_{t}^{\tau} r_{u} d u} \mid \mathcal{F}_{t}\right] .
$$

Furthermore inserting (2.15) and (2.16) in Proposition 2.3 gives the following expression for the short rate at at time $u, t \leq u \leq T_{i_{t}}+\widetilde{T}$ :

$$
\begin{aligned}
r_{u} & =f(t, u)+\int_{t}^{u} d f(s, u) \\
& =f(t, u)+\int_{t}^{u} \sigma(s, u) \int_{s}^{u} \sigma(s, v) d v d s+\int_{t}^{u} \sigma(s, u) d W_{s}^{Q} .
\end{aligned}
$$

Hence, since $\sigma$ is $\mathbb{F}$-adapted, we have that in addition to the present information $\mathcal{F}_{t}$ and the future development of $W^{Q}$ the future short rate at time $u, r_{u}$, may depend on the future outcome of $Y_{j}, j \in\left\{i_{t}+1, \ldots, i_{u}\right\}$. Thus, at a first glance it seems as if the expectation in (2.18) depends on the distribution of $Y_{j}, j \in\left\{i_{t}+1, \ldots, i_{\tau}\right\}$ under $Q$, such that the distribution of $Y_{j}, j \in\left\{i_{t}+1, \ldots, i_{T_{i_{t}}+\widetilde{T}}\right\}$ under $Q$ may be (partly) given at time $t$ by (2.18). However, as we shall see below, this is not the case. First observe that

$$
\begin{aligned}
\int_{t}^{\tau} \int_{t}^{u} \sigma(s, u) \int_{s}^{u} \sigma(s, v) d v d s d u & =\int_{t}^{\tau} \int_{t}^{u} \frac{1}{2} \frac{\partial}{\partial u}\left(\int_{s}^{u} \sigma(s, v) d v\right)^{2} d s d u \\
& =\frac{1}{2} \int_{t}^{\tau} \int_{s}^{\tau} \frac{\partial}{\partial u}\left(\int_{s}^{u} \sigma(s, v) d v\right)^{2} d u d s \\
& =\frac{1}{2} \int_{t}^{\tau}\left(\int_{s}^{\tau} \sigma(s, v) d v\right)^{2} d s
\end{aligned}
$$


and

$$
\int_{t}^{\tau} \int_{t}^{u} \sigma(s, u) d W_{s}^{Q} d u=\int_{t}^{\tau} \int_{s}^{\tau} \sigma(s, u) d u d W_{s}^{Q}
$$

Now, use that provided $\int_{s}^{\tau} \sigma(s, u) d u$ is sufficiently integrable it holds for fixed $\tau$ that

$$
E^{Q}\left[e^{-\frac{1}{2} \int_{t}^{\tau}\left(\int_{s}^{\tau} \sigma(s, u) d u\right)^{2} d s-\int_{t}^{\tau} \int_{s}^{\tau} \sigma(s, u) d u d W_{s}^{Q}} \mid \mathcal{F}_{t}\right]=1,
$$

such that

$$
E^{Q}\left[e^{-\int_{t}^{\tau} r_{u} d u} \mid \mathcal{F}_{t}\right]=e^{-\int_{t}^{\tau} f(t, u) d u} .
$$

Hence, no undesirable restrictions on the class of equivalent martingale measures occur when $\sigma$ is $\mathbb{F}$-adapted.

Since $\sigma$ is $\mathbb{F}$-adapted the volatility (and hence the drift under $Q$ ) of all forward rates at time $t$ may depend on $Y_{i}$ if $T_{i}<t$. Hence, the initial bond prices of the newly issued bonds at time $T_{i}$ may influence the future prices of not only the newly issued bonds, but also bonds with shorter time to maturity. As an alternative model consider the case where the dependence on $Y_{i}$ is restricted to the volatility of the forward rates (bond prices) introduced at time $T_{i}$ (and later). In this case the future development of the bond prices depend on the information at the time of issue and the future development of $W^{Q}$ only. As a last example we mention the (quite restrictive) case where $\sigma$ is $\mathbb{G}$-adapted, such that the information gathered from the issue of new bonds does not influence the volatility of the forward rates (bond prices).

\subsection{Trading in the bond market}

When trading in the extended bond market introduced in Section 2.2 two problems arise: Firstly, at any time infinitely many bonds are traded in the bond market and secondly, the bonds traded at time $t$ depend on the time considered. Regarding the first problem we note that since the forward rates are driven by a 1-dimensional Wiener process only, it is sufficient if we at all times are allowed to invest in two assets, which are not linearly dependent. Furthermore, we note that the second problem may be overcome by considering a new set of price processes defined for all $t$ including the same information as the original price processes. Thus, both problems are solved by considering the following two assets: A savings account with dynamics given by $(2.5)$ and an asset, with price process $X$, generated by investing 1 unit at time 0 and at time $t, 0 \leq t \leq \widehat{T}$, investing in the longest bond traded in the market. The dynamics of $X$ are given by

$$
d X_{t}=X_{t} \frac{d P\left(t, T_{i_{t}}+\widetilde{T}\right)}{P\left(t, T_{i_{t}}+\widetilde{T}\right)}, \quad X_{0}=1 .
$$

Inserting the bond price dynamics from (2.17) we get the following $Q$-dynamics of $X$

$$
d X_{t}=r_{t} X_{t} d t-\int_{t}^{T_{i_{t}}+\widetilde{T}} \sigma(t, u) d u X_{t} d W_{t}^{Q} .
$$

The price process $X$ can be seen as the best available approximation to the value process generated by a roll-over strategy in bonds with time to maturity $\widetilde{T}$. Such a value process 
is usually referred to as a rolling-horizon bond, see Rutkowski (1999). The idea of roll-over strategies is closely related to the Musiela parametrization of forward rates, see Musiela (1993), where the forward rates are parameterized by time to maturity instead of time of maturity. In a continuous-time setting where bonds with all maturities are traded, a rolling-horizon bond requires investments in infinitely many different bonds. However, here we only adjust the portfolio, when new bonds are issued, such that $X$ requires a finite number of bonds, $n+1$, only.

Following the ideas of Møller (2001b) we now define trading in the presence of payment processes. Henceforth fix an arbitrary equivalent martingale measure $Q$ for the model $(B, X, \mathbb{F})$, that is, we are working with the probability space $(\Omega, \mathcal{F}, Q)$ and the filtrations $\left(\mathbb{F}^{T_{i}}\right)_{i \in\{0, \ldots, n\}}$. We note that for all $i, i \in\{0, \ldots, n\}$, the discounted price process $X^{*}$ is a $\left(Q, \mathbb{F}^{T_{i}}\right)$-martingale. Here, and throughout the paper, we use an asterisk $\left(^{*}\right)$ to denote discounted price processes. Let $\left\langle X^{*}\right\rangle$ denote the predictable quadratic variation process for $X^{*}$ associated with $Q$ and $\mathbb{F}^{T_{n}}$, i.e. the unique predictable process such that $\left(X^{*}\right)^{2}-\left\langle X^{*}\right\rangle$ is a $\left(Q, \mathbb{F}^{T_{n}}\right)$-martingale. For any $i$ we now introduce the space $\mathcal{L}^{2}\left(Q_{X^{*}}, \mathbb{F}^{T_{i}}\right)$ of $\mathbb{F}^{T_{i}}$ predictable processes $\vartheta$ satisfying

$$
E^{Q}\left[\int_{0}^{\widehat{T}} \vartheta_{u}^{2} d\left\langle X^{*}\right\rangle_{u}\right]<\infty .
$$

An $\mathbb{F}^{T_{i}}$-trading strategy is any process $\varphi=(\vartheta, \eta)$, where $\vartheta \in \mathcal{L}^{2}\left(Q_{X^{*}}, \mathbb{F}^{T_{i}}\right)$ and $\eta$ is $\mathbb{F}^{T_{i}}$-adapted such that the value process $\mathcal{V}(\varphi)$ defined by

$$
\mathcal{V}_{t}(\varphi)=\vartheta_{t} X_{t}+\eta_{t} B_{t}, \quad 0 \leq t \leq \widehat{T},
$$

is RCLL (Right Continuous with Left Limits) and $\mathcal{V}_{t}(\varphi) \in \mathcal{L}^{2}(Q)$ for all $t \in[0, \widehat{T}]$. The pair $\varphi_{t}=\left(\vartheta_{t}, \eta_{t}\right)$ is interpreted as the portfolio held at time $t$. Here, $\vartheta$ denotes the number of assets with price process $X$, and $\eta$ denotes the discounted deposit in the savings account.

A payment process is an $\mathbb{F}$-adapted process $A=\left(A_{t}\right)_{0 \leq t \leq T}$ describing the liabilities of the seller of a contract towards the buyer. Note that $A$ is defined on $[0, T]$ only, such that no payments take place after time $T$. Moreover, we note that since $A$ is $\mathbb{F}$-adapted, it is $\mathbb{F}^{T_{i}}$-adapted for all $i \in\{1, \ldots, n\}$. We assume that $A$ is square integrable, i.e. that $E^{Q}\left[A_{t}^{2}\right]<\infty$ for all $t$, and RCLL. For $0 \leq s \leq t \leq T$, we let $A_{t}-A_{s}$ be the total outgoes less incomes in the interval $(s, t]$. In the following we shall consider the discounted payment process $A^{*}$ defined by

$$
d A_{t}^{*}=e^{-\int_{0}^{t} r_{u} d u} d A_{t} .
$$

The cost process associated with the pair $(\varphi, A)$ is given by

$$
C_{t}(\varphi)=\mathcal{V}_{t}^{*}(\varphi)-\int_{0}^{t} \vartheta_{u} d X_{u}^{*}+A_{t}^{*}
$$

Thus, the cost process is the discounted value of the portfolio reduced by discounted trading gains and added the total discounted outgoes less incomes of the payment process. The cost process is interpreted as the seller's accumulated discounted costs during $[0, t]$. The cost process is square integrable due to the square integrability of the payment process $A$ and the assumptions on the strategy $\varphi$ and $X$. Furthermore the cost process is adapted to the same filtration as the trading strategy. 
We say that a strategy $\varphi$ is $\mathbb{F}^{T_{i}}$-self-financing for the payment process $A$, if the cost process is constant $Q$-a.s. with respect to $\mathbb{F}^{T_{i}}$. In contrast to the classical definition of self-financing strategies, we thus allow for exogenous deposits and withdrawals as represented by $A$. The two definitions of self-financing strategies are equivalent if and only if the payment process is constant $Q$-a.s. with respect to the considered filtration. The interpretation of a selffinancing strategy in the presence of payment processes is that all fluctuations of the value process are either trading gains/losses or due to the payment process. A payment

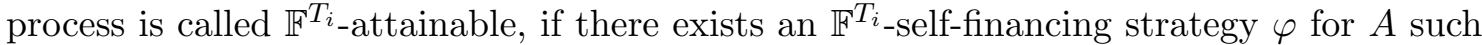
that $\mathcal{V}_{T}^{*}(\varphi)=0 Q$-a.s. with respect to $\mathbb{F}^{T_{i}}$. A payment process is thus $\mathbb{F}^{T_{i}}$-attainable, if investing the initial amount $C_{0}(\varphi)$ according to the trading strategy $\varphi$ leaves us with a portfolio value of 0 after the settlement of all liabilities. Hence, the unique arbitrage free price in $\left(B, X, \mathbb{F}^{T_{i}}\right)$ of an $\mathbb{F}^{T_{i}}$-attainable payment process is $C_{0}(\varphi)$. At any time $t$, there is no difference between receiving the future payments of the $\mathbb{F}^{T}{ }_{i}$-attainable payment process $A$ and holding the portfolio $\varphi_{t}$ and investing according to the $\mathbb{F}^{T_{i}}$-replicating strategy $\varphi$. Thus, a no arbitrage argument gives that at any time $t$ the price of future payments from $A$ in $\left(B, X, \mathbb{F}^{T_{i}}\right)$ must be $\mathcal{V}_{t}(\varphi)$. It can be shown that the payment process $A$ is attainable if and only if the contingent claim $H=A_{T}$ with maturity $T$ is (classically) attainable. If all contingent claims, and hence all payment processes, are attainable, the model is called complete and otherwise it is called incomplete.

\section{Risk-minimization}

As noted above, an $\mathbb{F}^{T_{i}}$-attainable payment process has a unique arbitrage free price $C_{0}(\varphi)$ in $\left(B, X, \mathbb{F}^{T_{i}}\right)$. However, for a non-attainable payment process, we do not have a unique arbitrage free price. Thus, for non-attainable processes, quantifying and controlling the risk becomes important. Here, we apply the criterion of risk-minimization. We give a review of risk-minimization and determine risk-minimizing strategies in the presence of reinvestment risk.

\subsection{A review of risk-minimization for payment processes}

In this section we review the concept of risk-minimization introduced by Föllmer and Sondermann (1986) for contingent claims, and further developed in Møller (2001b) to cover payment processes. For more details we refer to Møller (2001b). Throughout this section, we consider a fixed but arbitrary filtration $\mathbb{F}^{T_{i}}$, such that we are working with the filtered probability space $\left(\Omega, \mathcal{F}, Q, \mathbb{F}^{T_{i}}\right)$.

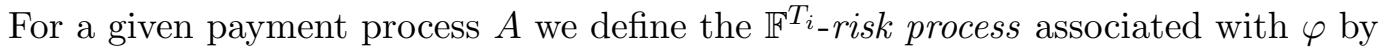

$$
R_{t}^{T_{i}}(\varphi)=E^{Q}\left[\left(C_{T}(\varphi)-C_{t}(\varphi)\right)^{2} \mid \mathcal{F}_{t}^{T_{i}}\right]
$$

where the cost process is defined in (2.20). Thus, the risk process is the conditional expectation of the discounted squared future costs given the current available information. We will use this quantity to measure the risk associated with $(\varphi, A)$. An $\mathbb{F}^{T_{i}}$-trading strategy $\varphi=(\vartheta, \eta)$ is called $\mathbb{F}^{T_{i}}$-risk-minimizing if for any $t \in[0, T]$ it minimizes $R_{t}^{T_{i}}(\varphi)$

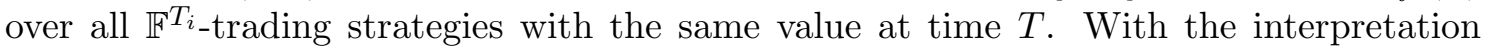


of the cost process in mind, we note that $\mathcal{V}_{t}^{*}(\varphi)$ is the discounted value of the portfolio $\varphi_{t}$ after possible payments at time $t$. In particular, $\mathcal{V}_{T}^{*}(\varphi)$ is the discounted value of the portfolio $\varphi_{T}$ upon settlement of all liabilities. Thus, a natural restriction is to consider so-called 0-admissible strategies which satisfy

$$
\mathcal{V}_{T}^{*}(\varphi)=0, \quad Q \text {-a.s. }
$$

The construction of risk-minimizing strategies is based on the so-called Galtchouk-KunitaWatanabe decomposition for martingales. Define the $\left(Q, \mathbb{F}^{T_{i}}\right)$-martingale $V^{T_{i}, *}$ by

$$
V_{t}^{T_{i}, *}=E^{Q}\left[A_{T}^{*} \mid \mathcal{F}_{t}^{T_{i}}\right], \quad 0 \leq t \leq T
$$

The process $V^{T_{i}, *}$, which is known as the intrinsic value process with respect to $\mathbb{F}^{T_{i}}$, can now be uniquely decomposed using the Galtchouk-Kunita-Watanabe decomposition

$$
V_{t}^{T_{i}, *}=V_{0}^{T_{i}, *}+\int_{0}^{t} \vartheta_{u}^{T_{i}, A} d X_{u}^{*}+L_{t}^{T_{i}, A} .
$$

Here, $L^{T_{i}, A}$ is a zero-mean square integrable $\left(Q, \mathbb{F}^{T_{i}}\right)$-martingale which is orthogonal to $X^{*}$, i.e. the process $X^{*} L^{T_{i}, A}$ is a $\left(Q, \mathbb{F}^{T_{i}}\right)$-martingale, and $\vartheta^{T_{i}, A}$ is an $\mathbb{F}^{T_{i}}$-predictable process in $\mathcal{L}^{2}\left(Q_{X^{*}}, \mathbb{F}^{T_{i}}\right)$. We note that if $A$ is $\mathbb{F}^{T_{i}}$-attainable, then $V_{t}^{T_{i}, *}$ is the discounted unique arbitrage free price in $\left(B, X, \mathbb{F}^{T_{i}}\right)$ at time $t$ of the future payments specified by the payment process $A$ and $L^{T_{i}, A}=0 Q$-a.s. with respect to $\mathbb{F}^{T_{i}}$. The following theorem relates the riskminimizing strategy and the associated risk process to the Galtchouk-Kunita-Watanabe decomposition.

\section{Theorem 3.1 (Møller (2001b))}

There exists a unique 0 -admissible $\mathbb{F}^{T_{i}}$-risk-minimizing strategy $\varphi^{T_{i}}=\left(\vartheta^{T_{i}}, \eta^{T_{i}}\right)$ for $A$ given by

$$
\left(\vartheta_{t}^{T_{i}}, \eta_{t}^{T_{i}}\right)=\left(\vartheta_{t}^{T_{i}, A}, V_{t}^{T_{i}, *}-A_{t}^{*}-\vartheta_{t}^{T_{i}, A} X_{t}^{*}\right), \quad 0 \leq t \leq T .
$$

The associated $\mathbb{F}^{T_{i}}$-risk process is given by

$$
R_{t}^{T_{i}}\left(\varphi^{T_{i}}\right)=E^{Q}\left[\left(L_{T}^{T_{i}, A}-L_{t}^{T_{i}, A}\right)^{2} \mid \mathcal{F}_{t}^{T_{i}}\right]
$$

When determining the risk-minimizing strategy, we minimize over all admissible strategies. This is in contrast to many other quadratic hedging criteria such as mean-variance indifference principles and mean-variance hedging, where only self-financing strategies are allowed. For more details on and a comparison of these criteria see Møller (2001a). As noted earlier, risk-minimizing strategies are not self-financing for non-attainable payment processes. However, they can be shown to be mean-self-financing, i.e. the corresponding cost processes are $Q$-martingales with respect to the considered filtration, see Møller (2001b, Lemma A.4).

Note that the risk-minimizing strategy depends on the choice of equivalent martingale measure $Q$. In the literature, the minimal martingale measure has been applied for determining risk-minimizing strategies, since this, in the case where $X^{*}$ is continuous, essentially corresponds to the criterion of local risk-minimization, which is a criterion in terms of $P$, see Schweizer (2001). 
Remark 3.2 We note that since $\mathcal{F}_{t}^{T_{i}}$ and $\mathcal{F}_{t}^{T_{j}}$ coincide for $t \geq \max \left(T_{j}, T_{i}\right)$ so do the intrinsic value processes. This is also intuitively clear, since for $t \geq \max \left(T_{j}, T_{i}\right)$ the additional information at time 0 in the larger of the filtrations has been revealed, and thus is included in the $\sigma$-algebra in the smaller filtration as well.

\subsection{Risk-minimization in the presence of reinvestment risk}

From Section 3.1 it follows that if we determine the Galtchouk-Kunita-Watanabe decomposition of $V^{T_{i}}$,* then the unique 0 -admissible $\mathbb{F}^{T_{i}}$-risk-minimizing strategy, $i \in\{0, \ldots, n\}$, is given by Theorem 3.1. However, since it is often difficult to determine the GaltchoukKunita-Watanabe decomposition we take a different approach. We apply the main result in Schweizer (1994) regarding risk-minimization under restricted information in order to obtain the following theorem, allowing us to determine the $\mathbb{F}^{T_{i}}$-risk-minimizing strategy in terms of the $\mathbb{F}^{T_{n}}$-risk-minimizing strategy.

Theorem 3.3

The unique 0-admissible $\mathbb{F}^{T_{i}}$-risk-minimizing strategy $\varphi^{T_{i}}=\left(\vartheta^{T_{i}}, \eta^{T_{i}}\right)$ for $A$ given by

$$
\left(\vartheta_{t}^{T_{i}}, \eta_{t}^{T_{i}}\right)=\left(E^{Q}\left[\vartheta_{t}^{T_{n}} \mid \mathcal{F}_{t-}^{T_{i}}\right], V_{t}^{T_{i}, *}-A_{t}^{*}-\vartheta_{t}^{T_{i}} X_{t}^{*}\right), \quad 0 \leq t \leq T,
$$

and the process $L^{T_{i}, A}$ is given by

$$
L_{t}^{T_{i}, A}=V_{0}^{T_{i} \vee T_{i_{t}}, *}-V_{0}^{T_{i}, *}+E^{Q}\left[\int_{0}^{t} \vartheta_{u}^{T_{n}} d X_{u}^{*} \mid \mathcal{F}_{t}^{T_{i}}\right]-\int_{0}^{t} \vartheta_{u}^{T_{i}} d X_{u}^{*} .
$$

Proof of Theorem 3.3: Since $\mathcal{F}_{t}^{T_{i}} \subseteq \mathcal{F}_{t}^{T_{n}}$ for all $t$ and $X^{*}$ is $\mathbb{F}$-adapted with $X_{T}^{*}$ being $\mathcal{F}_{T}^{T_{i}}$-measurable, Schweizer (1994, Theorem 3.1) gives the $\mathbb{F}^{T_{i}}$-risk-minimizing strategy for any $\mathcal{F}_{T}^{T_{i}}$-measurable contingent claim in terms of the $\mathbb{F}^{T_{n}}$-risk-minimizing strategy. Since both $X^{*}$ and $A$ are $\mathbb{F}^{T_{i}}$-adapted for all $i$, the result for contingent claims carries over to the present framework with payment processes. Using the fact that $X^{*}$ furthermore is $\mathbb{F}^{T_{i}}$ predictable for all $i$, we have from Schweizer (1994, Section 4), that the $\mathbb{F}^{T_{i}}$-risk-minimizing strategy is given by

$$
\vartheta_{t}^{T_{i}}=E^{Q}\left[\vartheta_{t}^{T_{n}} \mid \mathcal{F}_{t-}^{T_{i}}\right]
$$

and

$$
\begin{aligned}
\eta_{t}^{T_{i}} & =E^{Q}\left[V_{t}^{T_{n}, *}-A_{t}^{*}-\vartheta_{t}^{T_{i}} X_{t}^{*} \mid \mathcal{F}_{t}^{T_{i}}\right] \\
& =E^{Q}\left[V_{t}^{T_{n}, *} \mid \mathcal{F}_{t}^{T_{i}}\right]-A_{t}^{*}-\vartheta_{t}^{T_{i}} X_{t}^{*} \\
& =V_{t}^{T_{i}, *}-A_{t}^{*}-\vartheta_{t}^{T_{i}} X_{t}^{*} .
\end{aligned}
$$

Here, we have used that $\vartheta^{T_{i}}$ is $\mathbb{F}^{T_{i}}$-predictable, and that $A^{*}$ and $X^{*}$ are $\mathbb{F}^{T_{i}}$-adapted in the second equality, and iterated expectations in the third. To derive an expression for $L^{T_{i}, A}$ we first note that $V_{T}^{T_{n}, *}=V_{T}^{T_{i}, *}$, see Remark 3.2. Inserting the expressions from (3.3) and isolating $L_{T}^{T_{i}, A}$ we obtain

$$
L_{T}^{T_{i}, A}=V_{0}^{T_{n}, *}-V_{0}^{T_{i}, *}+\int_{0}^{T}\left(\vartheta_{u}^{T_{n}}-\vartheta_{u}^{T_{i}}\right) d X_{u}^{*}
$$


Using that $L^{T_{i}, A}$ is a $\left(Q, \mathbb{F}^{T_{i}}\right)$-martingale we get

$$
\begin{aligned}
L_{t}^{T_{i}, A} & =E^{Q}\left[L_{T}^{T_{i}, A} \mid \mathcal{F}_{t}^{T_{i}}\right] \\
& =E^{Q}\left[V_{0}^{T_{n}, *}-V_{0}^{T_{i}, *}+\int_{0}^{T}\left(\vartheta_{u}^{T_{n}}-\vartheta_{u}^{T_{i}}\right) d X_{u}^{*} \mid \mathcal{F}_{t}^{T_{i}}\right] \\
& =V_{0}^{T_{i} \vee T_{i_{t}}, *}-V_{0}^{T_{i}, *}+E^{Q}\left[\int_{0}^{t} \vartheta_{u}^{T_{n}} d X_{u}^{*} \mid \mathcal{F}_{t}^{T_{i}}\right]-\int_{0}^{t} \vartheta_{u}^{T_{i}} d X_{u}^{*} .
\end{aligned}
$$

Here, we have used that $X^{*}$ is a martingale and $\vartheta^{T_{j}}$ lies in $\mathcal{L}^{2}\left(Q_{X^{*}}, \mathbb{F}^{T_{j}}\right)$ such that the integral $E^{Q}\left[\int_{t}^{T} \vartheta_{u}^{T_{j}} d X_{u}^{*} \mid \mathcal{F}_{t}^{T_{i}}\right]=0$ for all $j$. Furthermore, we have used that $\mathcal{F}_{t}^{T_{i}}=\mathcal{F}_{t}^{T_{i} \vee T_{i_{t}}}$ to obtain

$$
E^{Q}\left[V_{0}^{T_{n}, *} \mid \mathcal{F}_{t}^{T_{i}}\right]=E^{Q}\left[V_{0}^{T_{n}, *} \mid \mathcal{F}_{t}^{T_{i} \vee T_{i_{t}}}\right]=E^{Q}\left[V_{0}^{T_{n}, *} \mid \mathcal{F}_{0}^{T_{i} \vee T_{i_{t}}}\right]=V_{0}^{T_{i} \vee T_{i_{t}}, *} .
$$

From Theorem 3.3 we get that the $\mathbb{F}^{T_{i}}$-risk-minimizing strategy is the predictable conditional expectation of the risk-minimizing strategy in the complete model $\left(B, X, \mathbb{F}^{T_{n}}\right)$ given the present information, $\mathcal{F}_{t}^{T_{i}}$. Thus, Theorem 3.3 provides an alternative to Theorem 3.1 when determining the $\mathbb{F}^{T_{i}}$-risk-minimizing strategy. The advantage of Theorem 3.3 is that, since $\left(B, X, \mathbb{F}^{T_{n}}\right)$ is a complete model, the $\mathbb{F}^{T_{n}}$-risk-minimizing strategy for any payment process coincides with the $\mathbb{F}^{T_{n}}$-replicating strategy. Hence, using Theorem 3.3 to determine the $\mathbb{F}^{T_{i}}$-risk-minimizing strategy requires the derivation of a replicating strategy in a complete model and a conditional expectation instead of the derivation of a Galtchouk-Kunita-Watanabe decomposition for a non-attainable payment process.

Remark 3.4 Investigating the risk minimizing strategies in Theorem 3.3 we observe that since $\mathcal{F}_{t}^{T_{i}}$ and $\mathcal{F}_{t}^{T_{j}}$ coincide for $t \geq \max \left(T_{i}, T_{j}\right)$, then the $\mathbb{F}^{T_{i}}$ and $\mathbb{F}^{T_{j}}$-risk-minimizing strategies coincide for $t>\max \left(T_{i}, T_{j}\right)$. The intuitive interpretation is that the strategies are based on the same information, and hence they are identical.

\section{Corollary 3.5}

If we restrict ourselves to payment processes for which $\vartheta^{T_{n}}$ is uniformly bounded then $L_{t}^{T_{i}, A}$ is given by

$$
L_{t}^{T_{i}, A}=V_{0}^{T_{i} \vee T_{i_{t}}, *}-V_{0}^{T_{i}, *}+\int_{0}^{t}\left(\vartheta_{u}^{T_{i} \vee T_{i_{t}}}-\vartheta_{u}^{T_{i}}\right) d X_{u}^{*}
$$

Proof of Corollary 3.5: Since $\vartheta^{T_{n}}$ is uniformly bounded we may use stochastic Fubini, see Protter (2004, Chapter IV, Theorem 64), to interchange the order of integration in (3.5).

The expression for $L^{T_{i}, A}$ in Corollary 3.5 has the following nice interpretation: At any time the unhedgeable part of $V^{T_{i}, *}$ consists of two terms. The first term is the difference between the initial deposit given the information at time 0 and the current information, respectively, whereas the second term is the difference between the trading gains generated by the risk-minimizing strategy given the present information regarding $Y$ and the $\mathbb{F}^{T_{i}}$ risk-minimizing strategy. In particular we note from Corollary 3.5 that for $t<T_{i+1}$, we have that $L_{t}^{T_{i}, A}=0$. This is also intuitively clear, since no additional information concerning the $Y_{j}$ 's has been revealed. 


\section{Corollary 3.6}

In the case where $\vartheta^{T_{n}}$ is uniformly bounded we have the following alternative expression for the process $L^{T_{i}, A}$ :

$$
L_{t}^{T_{i}, A}=\sum_{j=i+1}^{i_{t}}\left(V_{T_{j}}^{T_{j}, *}-E^{Q}\left[V_{T_{j}}^{T_{n}, *} \mid \mathcal{F}_{T_{j}-}^{T_{j-1}}\right]\right) .
$$

This leads to the following expression for the $\mathbb{F}^{T_{i}}$-risk process associated with $\varphi^{T_{i}}$

$$
R_{t}^{T_{i}}\left(\varphi^{T_{i}}\right)=E^{Q}\left[\left(\sum_{j=i_{t}+1}^{T}\left(V_{T_{j}}^{T_{j}, *}-E^{Q}\left[V_{T_{j}}^{T_{n}, *} \mid \mathcal{F}_{T_{j}-}^{T_{j-1}}\right]\right)\right)^{2} \mid \mathcal{F}_{t}^{T_{i}}\right] .
$$

Proof of Corollary 3.6: From Corollary 3.5 we have the following expression for $L_{t}^{T_{i}, A}$ :

$$
L_{t}^{T_{i}, A}=V_{0}^{T_{i} \vee T_{i_{t}}, *}-V_{0}^{T_{i}, *}+\int_{0}^{t}\left(\vartheta_{u}^{T_{i} \vee T_{i_{t}}}-\vartheta_{u}^{T_{i}}\right) d X_{u}^{*}
$$

Now, write $V_{0}^{T_{i} \vee T_{i_{t}}, *}-V_{0}^{T_{i}, *}$ and $\vartheta_{u}^{T_{i} \vee T_{i_{t}}}-\vartheta_{u}^{T_{i}}$ as telescoping sums and use that, as noted above, the $\mathbb{F}^{T_{i}}$ and $\mathbb{F}^{T_{j}}$-risk-minimizing strategies coincide for $t>\max \left(T_{i}, T_{j}\right)$ to obtain

$$
\begin{aligned}
L_{t}^{T_{i}, A} & =\sum_{j=i+1}^{i_{t}}\left(V_{0}^{T_{j}, *}-V_{0}^{T_{j-1}, *}+\int_{0}^{t}\left(\vartheta_{u}^{T_{j}}-\vartheta_{u}^{T_{j-1}}\right) d X_{u}^{*}\right) \\
& =\sum_{j=i+1}^{i_{t}}\left(V_{0}^{T_{j}, *}-V_{0}^{T_{j-1}, *}+\int_{0}^{T_{j}}\left(\vartheta_{u}^{T_{j}}-\vartheta_{u}^{T_{j-1}}\right) d X_{u}^{*}\right) .
\end{aligned}
$$

Using iterated expectations in order to express all quantities as expectations of the respective $\mathbb{F}^{T_{n}}$-quantities, we get

$$
\begin{aligned}
L_{t}^{T_{i}, A}= & \sum_{j=i+1}^{i_{t}}\left(E^{Q}\left[V_{0}^{T_{n}, *} \mid \mathcal{F}_{0}^{T_{j}}\right]-E^{Q}\left[V_{0}^{T_{n}, *} \mid \mathcal{F}_{0}^{T_{j-1}}\right]\right. \\
& \left.+\int_{0}^{T_{j}}\left(E^{Q}\left[\vartheta_{u}^{T_{n}} \mid \mathcal{F}_{u-}^{T_{j}}\right]-E^{Q}\left[\vartheta_{u}^{T_{n}} \mid \mathcal{F}_{u-}^{T_{j-1}}\right]\right) d X_{u}^{*}\right) .
\end{aligned}
$$

The result now follows from

$$
\begin{aligned}
L_{t}^{T_{i}, A}= & \sum_{j=i+1}^{i_{t}}\left(E^{Q}\left[V_{0}^{T_{n}, *} \mid \mathcal{F}_{T_{j}}^{T_{j}}\right]-E^{Q}\left[V_{0}^{T_{n}, *} \mid \mathcal{F}_{T_{j}-}^{T_{j-1}}\right]\right. \\
& \left.+\int_{0}^{T_{j}}\left(E^{Q}\left[\vartheta_{u}^{T_{n}} \mid \mathcal{F}_{T_{j}}^{T_{j}}\right]-E^{Q}\left[\vartheta_{u}^{T_{n}} \mid \mathcal{F}_{T_{j}-}^{T_{j-1}}\right]\right) d X_{u}^{*}\right) \\
= & \sum_{j=i+1}^{i_{t}}\left(E^{Q}\left[V_{0}^{T_{n}, *}+\int_{0}^{T_{j}} \vartheta_{u}^{T_{n}} d X_{u}^{*} \mid \mathcal{F}_{T_{j}}^{T_{j}}\right]-E^{Q}\left[V_{0}^{T_{n}, *}+\int_{0}^{T_{j}} \vartheta_{u}^{T_{n}} d X_{u}^{*} \mid \mathcal{F}_{T_{j}-}^{T_{j-1}}\right]\right) \\
= & \sum_{j=i+1}^{i_{t}}\left(E^{Q}\left[V_{T_{j}}^{T_{n}, *} \mid \mathcal{F}_{T_{j}}^{T_{j}}\right]-E^{Q}\left[V_{T_{j}}^{T_{n}, *} \mid \mathcal{F}_{T_{j}-}^{T_{i-1}}\right]\right) \\
= & \sum_{j=i+1}^{i_{t}}\left(V_{T_{j}}^{T_{j}, *}-E^{Q}\left[V_{T_{j}}^{T_{n}, *} \mid \mathcal{F}_{T_{j}-}^{T_{j-1}}\right]\right) .
\end{aligned}
$$


Here, we have used $E^{Q}\left[V_{0}^{T_{n}, *} \mid \mathcal{F}_{0}^{T_{j}}\right]=E^{Q}\left[V_{0}^{T_{n}, *} \mid \mathcal{F}_{t}^{T_{j}}\right]$ for $t<T_{j+1}$ and $E^{Q}\left[\vartheta_{u}^{T_{n}} \mid \mathcal{F}_{u-}^{T_{j}}\right]=$ $E^{Q}\left[\vartheta_{u}^{T_{n}} \mid \mathcal{F}_{t}^{T_{j}}\right]$ for $u \leq t<T_{j+1}$ in the first equality. The uniform boundedness of $\vartheta^{T_{n}}$ allows us to use stochastic Fubini, see Protter (2004, Chapter IV, Theorem 64), to interchange the order of integration in the second equality. Furthermore we have used that $\varphi^{T_{n}}$ is self-financing in the third equality and the definition of $V^{T_{j}, *}$ in the last equality. The expression for the $\mathbb{F}^{T_{i}}$-risk process associated with $\varphi^{T_{i}}$ in (3.6) is now obtained by inserting the expression for $L^{T_{i}, A}$ in (3.4).

From Corollary 3.6 we observe that $L^{T_{i}, A}$, which measures the deposits or withdrawals to/from the risk-minimizing portfolio in addition to those generated by the payment process, only changes value at times $T_{j}, j>i$. Hence, the risk-minimizing strategy is self-financing between the times of issue. At time $T_{j}$ the information revealed by the issued bonds, i.e. the observed value of $Y_{j}$, affects the weights given to the different outcomes of $Y$, and hence it leads to a change in $L^{T_{i}, A}$.

\section{3 $\quad \mathbb{F}$-risk-minimizing strategies}

We now derive the $\mathbb{F}$-risk-minimizing strategy for a general payment process of the form

$$
d A_{t}=\Delta A_{0} d 1_{(t \geq 0)}+a_{t} d t+\Delta A_{T} d 1_{(t \geq T)} .
$$

Here, $\Delta A_{0}$ is a constant, whereas $a_{t}$ is $\mathcal{F}_{t}$-measurable for all $t$, and $\Delta A_{T}$ is $\mathcal{F}_{T}$-measurable. We note that the payment process is $\mathbb{F}$ - and $\mathbb{F}^{T_{n}}$-adapted. In order to derive the riskminimizing strategy we consider the discounted payment process

$$
A_{t}^{*}=A_{0}+\int_{0}^{t} e^{-\int_{0}^{s} r_{u} d u} d A_{s}=A_{0}+\int_{0}^{t} e^{-\int_{0}^{s} r_{u} d u} a_{s} d s+e^{-\int_{0}^{T} r_{s} d s} \Delta A_{T} 1_{(t=T)} .
$$

Since the model $\left(B, X, \mathbb{F}^{T_{n}}\right)$ is complete, we have the following expression for $A^{*}$ :

$$
A_{t}^{*}=A_{0}+\int_{0}^{t}\left(F_{0}^{T_{n}, s}+\int_{0}^{s} \vartheta_{u}^{T_{n}, s} d X_{u}^{*}\right) d s+\left(F_{0}^{T_{n}, \Delta T}+\int_{0}^{T} \vartheta_{u}^{T_{n}, \Delta T} d X_{u}^{*}\right) 1_{(t=T)},
$$

where $\left(\vartheta^{T_{n}, s}\right)_{0 \leq s \leq T}$ and $\vartheta^{T_{n}, \Delta T}$ are the replicating strategies in $\left(B, X, \mathbb{F}^{T_{n}}\right)$ for $\left(a_{s}\right)_{0 \leq s \leq T}$ and $\Delta A_{T}$, respectively, and we for $0 \leq t \leq s \leq T$ have defined

$$
F_{t}^{T_{n}, s}=E^{Q}\left[e^{-\int_{t}^{s} r_{u} d u} a_{s} \mid \mathcal{F}_{t}^{T_{n}}\right] \text { and } F_{t}^{T_{n}, \Delta T}=E^{Q}\left[e^{-\int_{t}^{T} r_{u} d u} \Delta A_{T} \mid \mathcal{F}_{t}^{T_{n}}\right] .
$$

Hence, $F_{t}^{T_{n}, s}$ and $F_{t}^{T_{n}, \Delta T}$ are the unique arbitrage free prices at time $t$ in the model $\left(B, X, \mathbb{F}^{T_{n}}\right)$ for the claims $a_{s}$ and $\Delta A_{T}$, respectively. Now use that $\left(B, X, \mathbb{F}^{T_{n}}\right)$ is complete to obtain

$$
\begin{aligned}
V_{t}^{T_{n}, *} & =E^{Q}\left[A_{T}^{*} \mid \mathcal{F}_{t}^{T_{n}}\right] \\
& =E^{Q}\left[A_{0}+\int_{0}^{T}\left(F_{0}^{T_{n}, s}+\int_{0}^{s} \vartheta_{u}^{T_{n}, s} d X_{u}^{*}\right) d s+\left(F_{0}^{T_{n}, \Delta T}+\int_{0}^{T} \vartheta_{u}^{T_{n}, \Delta T} d X_{u}^{*}\right) \mid \mathcal{F}_{t}^{T_{n}}\right] \\
& =A_{0}+\int_{0}^{T} F_{0}^{T_{n}, s} d s+F_{0}^{T_{n}, \Delta T}+E^{Q}\left[\int_{0}^{T} \int_{0}^{s} \vartheta_{u}^{T_{n}, s} d X_{u}^{*} d s+\int_{0}^{T} \vartheta_{u}^{T_{n}, \Delta T} d X_{u}^{*} \mid \mathcal{F}_{t}^{T_{n}}\right] .
\end{aligned}
$$


Here, we restrict ourselves to payment processes for which $\left(\vartheta^{T_{n}, s}\right)_{0 \leq s \leq T}$ are uniformly bounded, such that we may use stochastic Fubini, see Protter (2004, Chapter IV, Theorem 64), to interchange the order of integration above. Hence, we obtain the following Galtchouk-Kunita-Watanabe decomposition of $V^{T_{n}, *}$ :

$$
\begin{aligned}
V_{t}^{T_{n}, *} & =V_{0}^{T_{n}, *}+E^{Q}\left[\int_{0}^{T} \int_{u}^{T} \vartheta_{u}^{T_{n}, s} d s d X_{u}^{*}+\int_{0}^{T} \vartheta_{u}^{T_{n}, \Delta T} d X_{u}^{*} \mid \mathcal{F}_{t}^{T_{n}}\right] \\
& =V_{0}^{T_{n}, *}+\int_{0}^{t} \int_{u}^{T} \vartheta_{u}^{T_{n}, s} d s d X_{u}^{*}+\int_{0}^{t} \vartheta_{u}^{T_{n}, \Delta T} d X_{u}^{*} \\
& =V_{0}^{T_{n}, *}+\int_{0}^{t} \vartheta_{u}^{T_{n}, A} d X_{u}^{*},
\end{aligned}
$$

where

$$
\vartheta_{u}^{T_{n}, A}=\int_{u}^{T} \vartheta_{u}^{T_{n}, s} d s+\vartheta_{u}^{T_{n}, \Delta T}
$$

Recall that $\vartheta^{T_{n}}=\vartheta^{T_{n}, A}$. The $\mathbb{F}$-risk-minimizing strategy $\varphi^{0}=\left(\vartheta^{0}, \eta^{0}\right)$ and the associated risk process are now given by inserting (3.7) in Theorem 3.3.

\subsection{1 $\quad$ F-risk-minimizing strategies when $Y$ has finite support}

Consider the case where $Y_{i}, i \in\{1, \ldots, n\}$, has finite support, hence $Y_{i} \in\left\{y_{1}^{i}, \ldots, y_{m_{i}}^{i}\right\}$. Let $K=\prod_{i=1}^{n} m_{i}$ denote the possible number of outcomes of the vector $Y$. To simplify the expression for the risk-minimizing strategies we introduce the notation

$$
M_{t}^{\delta_{k}}=E^{Q}\left[1_{\left(\left(Y_{1}, \ldots, Y_{n}\right)=\delta_{k}\right)} \mid \mathcal{F}_{t}\right]=E^{Q}\left[1_{\left(\left(Y_{1}, \ldots, Y_{n}\right)=\delta_{k}\right)} \mid \mathcal{H}_{t}\right],
$$

where $\delta_{1}, \ldots, \delta_{K}$ are the possible outcomes of the vector $\left(Y_{1}, \ldots, Y_{n}\right)$. Here, we have used the $Q$-independence between $Y$ and $W^{Q}$ in the second equality. If we further introduce the notation $\vartheta^{\delta_{k}}$ and $V^{\delta_{k}, *}$ to denote, respectively, the replicating strategy and the intrinsic value process given $Y=\delta_{k}$, then the $\mathbb{F}$-risk-minimizing strategy is given by

$$
\left(\vartheta_{t}^{0}, \eta_{t}^{0}\right)=\left(\sum_{k=1}^{K} M_{t-}^{\delta_{k}} \vartheta_{t}^{\delta_{k}}, \sum_{k=1}^{K} M_{t}^{\delta_{k}} V_{t}^{\delta_{k}, *}-A_{t}^{*}-\vartheta_{t}^{0} X_{t}^{*}\right), 0 \leq t \leq T .
$$

In this case the expression for the process $L^{0, A}$ in Corollary 3.6 simplifies to

$$
\begin{aligned}
L_{t}^{0, A} & =\sum_{j=1}^{i_{t}}\left(V_{T_{j}, *}^{T_{j},}-E^{Q}\left[V_{T_{j}}^{T_{n}, *} \mid \mathcal{F}_{T_{j}-}^{T_{j-1}}\right]\right) \\
& =\sum_{j=1}^{i_{t}}\left(E^{Q}\left[V_{T_{j}}^{T_{n}, *} \mid \mathcal{F}_{T_{j}}^{T_{j}}\right]-E^{Q}\left[V_{T_{j}}^{T_{n}, *} \mid \mathcal{F}_{T_{j}-}^{T_{j-1}}\right]\right) \\
& =\sum_{j=1}^{i_{t}}\left(\sum_{k=1}^{K} M_{T_{j}}^{\delta_{k}} V_{T_{j}}^{\delta_{k}, *}-\sum_{k=1}^{K} M_{T_{j-1}}^{\delta_{k}} V_{T_{j}}^{\delta_{k}, *}\right) \\
& =\sum_{j=1}^{i_{t}} \sum_{k=1}^{K} V_{T_{j}, *}^{\delta_{k},}\left(M_{T_{j}}^{\delta_{k}}-M_{T_{j-1}}^{\delta_{k}}\right) \\
& =\sum_{k=1}^{K} \int_{0}^{t} V_{u}^{\delta_{k}, *} d M_{u}^{\delta_{k}} .
\end{aligned}
$$


Here, we have used that the probabilities change at times $T_{i}, i=1, \ldots, n$, only, in the last equation, and that we are allowed to interchange summation and integration. Hence, in the case where $Y$ has finite support we have the following simple Galtchouk-KunitaWatanabe decomposition:

$$
V_{t}^{0, *}=\sum_{k=1}^{K} M_{0}^{\delta_{k}} V_{0}^{\delta_{k}, *}+\int_{0}^{t} \sum_{k=1}^{K} M_{u-}^{\delta_{k}} \vartheta_{u}^{\delta_{k}} d X_{u}^{*}+\sum_{k=1}^{K} \int_{0}^{t} V_{u}^{\delta_{k}, *} d M_{u}^{\delta_{k}} .
$$

Example 3.7 Consider the case where $Y=Y_{1}$ follows a binomial distribution, i.e. $Y \in$ $\{0,1\}$ with $1-P(Y=0)=P(Y=1)=p, p \in(0,1)$. Now the goal is to determine the risk-minimizing strategy under the minimal martingale measure, $Q^{0}$. In this simple example with just two possible outcomes of $Y$ we have $\delta_{i}=i-1, i \in\{1,2\}$. We do not specify the payment process, the forward rate dynamics and $\gamma$. Here, the quantities $M^{\delta_{k}}$ simplify to

$$
\begin{aligned}
& M_{t}^{\delta_{1}}=E^{Q^{0}}\left[1_{\left(Y=\delta_{1}\right)} \mid \mathcal{F}_{t}\right]=(1-p) 1_{\left(0 \leq t<T_{1}\right)}+(1-Y) 1_{\left(T_{1} \leq t \leq T\right)}, \\
& M_{t}^{\delta_{2}}=E^{Q^{0}}\left[1_{\left(Y=\delta_{2}\right)} \mid \mathcal{F}_{t}\right]=p 1_{\left(0 \leq t<T_{1}\right)}+Y 1_{\left(T_{1} \leq t \leq T\right)},
\end{aligned}
$$

where we have used that the distribution of $Y$ is unaffected by the change to the minimal martingale measure. Furthermore, the intrinsic value process $V^{0, *}$ given by

$$
V_{t}^{0, *}=1_{\left(0 \leq t<T_{1}\right)}\left((1-p) V_{t}^{\delta_{1}, *}+p V_{t}^{\delta_{2}, *}\right)+1_{\left(T_{1} \leq t \leq T\right)}\left((1-Y) V_{t}^{\delta_{1}, *}+Y V_{t}^{\delta_{2}, *}\right) .
$$

Inserting (3.10) and (3.11) into (3.8) gives the following risk-minimizing strategy

$$
\vartheta_{t}^{0}=1_{\left(0 \leq t \leq T_{1}\right)}\left((1-p) \vartheta_{t}^{\delta_{1}}+p \vartheta_{t}^{\delta_{2}}\right)+1_{\left(T_{1}<t \leq T\right)}\left((1-Y) \vartheta_{t}^{\delta_{1}}+Y \vartheta_{t}^{\delta_{2}}\right),
$$

and

$$
\eta_{t}^{0}=V_{t}^{0, *}-A_{t}^{*}-\left(1_{\left(0 \leq t \leq T_{1}\right)}\left((1-p) \vartheta_{t}^{\delta_{1}}+p \vartheta_{t}^{\delta_{2}}\right)+1_{\left(T_{1}<t \leq T\right)}\left((1-Y) \vartheta_{t}^{\delta_{1}}+Y \vartheta_{t}^{\delta_{2}}\right)\right) X_{t}^{*}
$$

where $V_{t}^{0, *}$ is given by (3.12). Now, inserting (3.10)-(3.13) in (3.9) gives the following Galtchouk-Kunita-Watanabe decomposition

$$
\begin{aligned}
V_{t}^{0, *}= & (1-p) V_{0}^{\delta_{1}, *}+p V_{0}^{\delta_{2}, *}+\int_{0}^{t}\left(1_{\left(0 \leq u \leq T_{1}\right)}\left((1-p) \vartheta_{u}^{\delta_{1}}+p \vartheta_{u}^{\delta_{2}}\right)\right. \\
& \left.+1_{\left(T_{1}<u \leq T\right)}\left((1-Y) \vartheta_{u}^{\delta_{1}}+Y \vartheta_{u}^{\delta_{2}}\right)\right) d X_{u}^{*}+1_{\left(T_{1} \leq t \leq T\right)}(Y-p)\left(V_{T_{1}, *}^{\delta_{2}}-V_{T_{1}}^{\delta_{1}, *}\right) .
\end{aligned}
$$

Example 3.8 We now extend Example 3.7 by specifying the payment process, the forward rate dynamics and the function $\gamma$. Hence, we still assume that $Y=Y_{1} \in\{0,1\}$ with $1-P(Y=0)=P(Y=1)=p, p \in(0,1)$. Consider a company, which at time 0 wants to hedge a claim of 1 at time $T$, i.e. $\Delta A_{T}=1$. Without loss of generality we assume $T=\widetilde{T}+T_{1}$. To model the dynamics of the forward rates, we let $\sigma$ be given by

$$
\sigma(t, \tau)=c e^{-a(\tau-t)},
$$

for some positive constants $c$ and $a$. Here, as in practice, fluctuations of the forward rates dampen exponentially as a function of time to maturity. Using that

$$
\int_{t}^{\tau} \sigma(t, u) d u=\int_{t}^{\tau} c e^{-a(u-t)} d u=\frac{c}{a}\left(1-e^{-a(\tau-t)}\right)
$$


we obtain the following forward rate dynamics under $Q$ for $0 \leq t \leq \tau \leq T_{i_{t}}+\widetilde{T}$ :

$$
d f(t, \tau)=\frac{c^{2}}{a} e^{-a(\tau-t)}\left(1-e^{-a(\tau-t)}\right) d t+c e^{-a(\tau-t)} d W_{t}^{Q} .
$$

To model the extension of the forward rate curve at time $T_{1}$ we assume $\gamma$ is given by

$$
\gamma_{s}=\frac{1}{T-\widetilde{T}}\left(k_{1} Y+k_{2}(1-Y)\right),
$$

for some constants $k_{1}$ and $k_{2}$. Thus, the forward rate curve is continued by a straight line with slope $k_{1} /(T-\widetilde{T})$ or $k_{2} /(T-\widetilde{T})$. In order to obtain the $\mathbb{F}$-risk-minimizing strategy under $Q^{0}$ we now consider the complete model $\left(B, S, \mathbb{F}^{T_{1}}\right)$, where $Y$ is known. Proposition 2.3 gives the following expression for the short rate

$$
r_{t}= \begin{cases}f(0, t)+\int_{0}^{t} d f(s, t), & t \leq \widetilde{T} \\ f(0, \widetilde{T})+\int_{0}^{T_{1}} d f(s, \widetilde{T})+\int_{\widetilde{T}}^{t} \gamma_{s} d s+\int_{T_{1}}^{t} d f(s, t), & t>\widetilde{T}\end{cases}
$$

We note from (3.16) that $r_{t}$ depends on $Y$ for $t>\widetilde{T}$. Inserting (3.14) and (3.15) in (3.16) gives

$$
r_{t}= \begin{cases}m(t)+\int_{0}^{t} c e^{-a(t-s)} d W_{s}^{Q}, & t \leq \widetilde{T} \\ m(t, Y)+\int_{T_{1}}^{t} c e^{-a(t-s)} d W_{s}^{Q}, & t>\widetilde{T},\end{cases}
$$

where we have defined

$$
m(t)=f(0, t)+\frac{c^{2}}{2 a^{2}}\left(1-e^{-a t}\right)^{2}, t \leq \widetilde{T},
$$

and

$$
\begin{aligned}
m(t, Y)= & f(0, \widetilde{T})+\frac{c^{2}}{2 a^{2}}\left(\left(1-e^{-a \widetilde{T}}\right)^{2}-\left(1-e^{-a\left(\widetilde{T}-T_{1}\right)}\right)^{2}+\left(1-e^{-a\left(t-T_{1}\right)}\right)^{2}\right) \\
& +\frac{t-\widetilde{T}}{T-\widetilde{T}}\left(k_{1} Y+k_{2}(1-Y)\right)+\int_{0}^{T_{1}} c e^{-a(\widetilde{T}-s)} d W_{s}^{Q}, \quad t>\widetilde{T} .
\end{aligned}
$$

Using Itô's formula we now obtain the short rate dynamics

$$
d r_{t}= \begin{cases}\left(\phi(t)-a r_{t}\right) d t+c d W_{t}^{Q}, & t \leq \widetilde{T} \\ \left(\phi(t, Y)-a r_{t}\right) d t+c d W_{t}^{Q}, & t>\widetilde{T}\end{cases}
$$

where

$$
\phi(t)=a m(t)+\frac{\partial}{\partial t} m(t) \text { and } \phi(t, Y)=a m(t, Y)+\frac{\partial}{\partial t} m(t, Y)
$$

Hence, given $Y$ the short rate follows an extended Vasiček model under $Q$. The result is well-known for $t \leq \widetilde{T}$, where $r_{t}$ is independent of $Y$, see e.g. Musiela and Rutkowski (1997). From (3.17) we observe that the drift and the squared diffusion both are affine in $r$, such that an extended Vasiček model for the short rate leads to an affine term structure, see e.g. Björk (2004, Proposition 22.2). Thus, in the conditional model we have the following expression for the unique arbitrage free price at time $t$ for 1 unit at time $T$ :

$$
P^{\delta_{Y+1}}(t, T)=\exp \left(A(t, T, Y)-B(t, T) r_{t}\right)
$$


with $A(t, T, Y)$ and $B(t, T)$ given by

$$
\begin{aligned}
B(t, T) & =\frac{1}{a}\left(1-e^{-a(T-t)}\right), \\
A(t, T, Y) & =\int_{t}^{T} \frac{1}{2} c^{2} B^{2}(s, T) d s-\int_{t}^{\widetilde{T}} \phi(s) B(s, T) d s-\int_{\widetilde{T}}^{T} \phi(s, Y) B(s, T) d s .
\end{aligned}
$$

Even though $B$ in general is allowed to depend on $Y$, it is not the case here, so we have omitted $Y$ in the notation for $B$. Note that we have used the notation $P^{\delta_{Y+1}}(t, T)$ even though the bond is not traded. Applying Itô's formula to (3.18) and using the differential equations for $A$ and $B$ from Björk (2004, Proposition 22.2), we obtain the following $Q$ dynamics for the price process $P^{\delta_{Y+1}}(t, T)$ :

$$
d P^{\delta_{Y+1}}(t, T)=r_{t} P^{\delta_{Y+1}}(t, T) d t-c B(t, T) P^{\delta_{Y+1}}(t, T) d W_{t}^{Q} .
$$

Combining (2.19), (3.19) and (3.20) gives

$$
d X_{t}=r_{t} X_{t} d t-c B\left(t, T_{i_{t}}+\widetilde{T}\right) X_{t} d W_{t}^{Q} .
$$

Note that at time $t, t>\widetilde{T}$, we have that $X_{t}$ depends on $Y$ through the short rate process. However, if we consider the discounted price process, $X^{*}$, the dynamics are given by

$$
d X_{t}^{*}=-c B\left(t, T_{i_{t}}+\widetilde{T}\right) X_{t}^{*} d W_{t}^{Q},
$$

such that $X^{*}$ is independent of $Y$. Comparing (3.20) and (3.21) we find that given $Y$ the replicating strategy is given by

$$
\vartheta_{t}^{\delta_{Y+1}}=\frac{B(t, T) P^{\delta_{Y+1}}(t, T)}{B\left(t, T_{i_{t}}+\widetilde{T}\right) X_{t}}=\frac{B(t, T) P^{\delta_{Y+1}, *}(t, T)}{B\left(t, T_{i_{t}}+\widetilde{T}\right) X_{t}^{*}} .
$$

Since

$$
V_{t}^{\delta_{Y+1}, *}=e^{-\int_{0}^{t} r_{u} d u} P^{\delta_{Y+1}}(t, T)=P^{\delta_{Y+1}, *}(t, T),
$$

we have

$$
\begin{aligned}
V_{t}^{0, *}= & 1_{\left(0 \leq t<T_{1}\right)}\left((1-p) P^{\delta_{1}, *}(t, T)+p P^{\delta_{2}, *}(t, T)\right) \\
& +1_{\left(T_{1} \leq t \leq T\right)}\left((1-Y) P^{\delta_{1}, *}(t, T)+Y P^{\delta_{2}, *}(t, T)\right) .
\end{aligned}
$$

Inserting (3.22) and (3.23) in the results from Example 3.7 gives the following riskminimizing strategy:

$$
\begin{aligned}
\vartheta_{t}^{0}= & \frac{1}{X_{t}^{*}}\left(1_{\left(0 \leq t \leq T_{1}\right)} \frac{B(t, T)}{B\left(t, T_{i_{t}}+\widetilde{T}\right)}\left((1-p) P^{\delta_{1}, *}(t, T)+p P^{\delta_{2}, *}(t, T)\right)\right. \\
& \left.+1_{\left(T_{1}<t \leq T\right)}\left((1-Y) P^{\delta_{1}, *}(t, T)+Y P^{\delta_{2}, *}(t, T)\right)\right)
\end{aligned}
$$

and

$$
\eta_{t}^{0}= \begin{cases}(1-p) P^{\delta_{1}, *}(t, T)+p P^{\delta_{2}, *}(t, T)-\left((1-p) \vartheta_{t}^{\delta_{1}}+p \vartheta_{t}^{\delta_{2}}\right) X_{t}^{*}, & 0<t<T_{1}, \\ (1-Y) P^{\delta_{1}, *}(t, T)+Y P^{\delta_{2}, *}(t, T)-\left((1-p) \vartheta_{t}^{\delta_{1}}+p \vartheta_{t}^{\delta_{2}}\right) X_{t}^{*}, & t=T_{1}, \\ 0, & T_{1}<t \leq T\end{cases}
$$


Investigating (3.24) and (3.25) we note that for $t>T_{1}$ the risk-minimizing strategy consists of $P^{\delta_{Y+1}, *}(t, T) / X_{t}^{*}$ units of the risky asset, which at this time corresponds to investing in bonds with maturity $T$. Hence, holding $P^{\delta_{Y+1}, *}(t, T) / X_{t}^{*}$ units of the risky asset is equivalent to holding one bond with maturity $T$, which in turn is the replicating strategy. The Galtchouk-Kunita-Watanabe decomposition is given by

$$
\begin{aligned}
V_{t}^{0, *}= & (1-p) P^{\delta_{1}, *}(0, T)+p P^{\delta_{2}, *}(0, T) \\
& +\int_{0}^{t} \frac{1}{X_{u}^{*}}\left(1_{\left(0 \leq u \leq T_{1}\right)} \frac{B(u, T)}{B\left(u, T_{i_{u}}+\widetilde{T}\right)}\left((1-p) P^{\delta_{1}, *}(u, T)+p P^{\delta_{2}, *}(u, T)\right)\right. \\
& \left.+1_{\left(T_{1}<u \leq T\right)}\left((1-Y) P^{\delta_{1}, *}(u, T)+Y P^{\delta_{2}, *}(u, T)\right)\right) d X_{u}^{*} \\
& +1_{\left(T_{1} \leq t \leq T\right)}(Y-p)\left(P^{\delta_{2}, *}\left(T_{1}, T\right)-P^{\delta_{1}, *}\left(T_{1}, T\right)\right) .
\end{aligned}
$$

\section{A practical implementation of the model}

In this section we discuss a possible implementation of the model. Without loss of generality we assume that new bonds are issued at time 0 , such that at time 0 the time to maturity of the longest traded bond is $\widetilde{T}$.

At time 0 we observe the bond prices in the market. Assuming the forward rates are given by a parametric model, with parameter $\theta \in \Theta$, we estimate the value of $\theta$, say $\theta_{0}$, which gives the best correspondence with the observed bond prices. For a possible parametrization we refer to Svensson (1995), who considers an extension of the so-called Nelson-Siegel parametrization; see Nelson and Siegel (1987) for the original Nelson-Siegel parametrization. Now let the initial forward rate curve at time $0,(f(0, \tau))_{0 \leq \tau \leq \widetilde{T}}$, be given by the estimated forward rate curve $\left(f^{\theta_{0}}(0, \tau)\right)_{0 \leq \tau<\widetilde{T}}$. In addition to the initial forward rate curve we, for later purpose, use $\theta_{0}$ to estimate $f^{\theta_{0}}\left(0, T_{1}+\widetilde{T}\right)$. Given a model for the forward rate dynamics we simulate the forward rate vector $\left(f\left(T_{1}, \tau\right)\right)_{T_{1} \leq \tau \leq \widetilde{T}}$ and the point $f^{\theta_{0}}\left(T_{1}, T_{1}+\widetilde{T}\right)$. The forward rate $f\left(T_{1}, T_{1}+\widetilde{T}\right)$ is now drawn from a distribution (estimated from historical data) with mean $f^{\theta_{0}}\left(T_{1}, T_{1}+\widetilde{T}\right)$. To obtain the forward rate curve at time $T_{1}$ after the issue of new bonds we combine $f\left(T_{1}, \widetilde{T}\right)$ and $f\left(T_{1}, T_{1}+\widetilde{T}\right)$ by a method giving a smooth extension of the forward rate curve. One possibility is the method of cubic splines, see e.g. Press, Flannery, Teukolsky and Vetterling (1986). Using the parametric forward rate model, we now estimate the parameter $\theta_{1}$, which gives the best correspondence with the forward rate curve at time $T_{1}$. The estimated parameter is only used to estimate $f^{\theta_{1}}\left(T_{1}, T_{2}+\widetilde{T}\right)$. Starting from the forward rate curve at time $T_{1}$, $\left(f\left(T_{1}, T_{1}+\tau\right)\right)_{0 \leq \tau \leq \widetilde{T}}$, and $f^{\theta_{1}}\left(T_{1}, T_{2}+\widetilde{T}\right)$ the procedure above is repeated to determine the forward rate curve at time $T_{2}$, and in turn the forward rate curve at any future time.

When implementing the model as described above we have the standard problems of estimating the initial forward rate curve from the observed bond prices and modelling the forward rates. In addition we have the model related problem of determining the distribution of $f\left(T_{i}, T_{i}+\widetilde{T}\right), i \in\{1, \ldots, n\}$. We note, however, that we avoid a direct modelling of $\gamma^{i}$. Instead $\gamma^{i}$ is given indirectly by $f^{\theta_{i-1}}\left(T_{i}, T_{i}+\widetilde{T}\right)$, the estimated distribution with mean $f^{\theta_{i-1}}\left(T_{i}, T_{i}+\widetilde{T}\right)$ and the chosen smoothing method. 


\section{Acknowledgements}

Financial support from Codan Pension, Danica Pension, Nordea Pension, Pen-Sam, PFA Pension and PKA is gratefully acknowledged. Furthermore, the author thanks supervisors Mogens Steffensen and Thomas Møller, as well as Rolf Poulsen for helpful comments and fruitful discussions. Also comments from Peter Holm Nielsen are appreciated.

\section{References}

Björk, T. (2004). Arbitrage Theory in Continuous Time, 2nd edn, Oxford University Press.

Björk, T., Kabanov, Y. and Runggaldier, W. (1997). Bond Market Structure in the Presence of Marked Point Processes, Mathematical Finance 7, 211-239.

Dahl, M. (2005). A Discrete-Time Model for Reinvestment Risk in Bond Markets, Working paper, Laboratory of Actuarial Mathematics, University of Copenhagen.

Föllmer, H. and Sondermann, D. (1986). Hedging of Non-Redundant Contingent Claims, in W. Hildenbrand and A. Mas-Colell (eds), Contributions to Mathematical Economics, NorthHolland, 205-223.

Heath, D., Jarrow, R. and Morton, A. (1992). Bond Pricing and the Term Structure of Interest Rates, Econometrica 60(1), 77-106.

Møller, T. (2001a). On Transformations of Actuarial Valuation Principles, Insurance: Mathematics and Economics 28, 281-303.

Møller, T. (2001b). Risk-Minimizing Hedging Strategies for Insurance Payment Processes, Finance and Stochastics 5(4), 419-446.

Musiela, M. (1993). Stochastic PDEs and Term Structure Models, Journées Internationales de Finance IGR-AFFI. La Baule, June 1993.

Musiela, M. and Rutkowski, M. (1997). Martingale Methods in Financial Modelling, SpringerVerlag, Berlin.

Nelson, C. R. and Siegel, A. F. (1987). Parsimonious Modeling of Yield Curves, Journal of Business 60(4), 473-489.

Neuberger, A. (1999). Hedging Long-Term Exposures with Multiple Short-Term Futures Contracts, The Review of Financial Studies 12(3), 429-459.

Press, W. H., Flannery, B. P., Teukolsky, S. A. and Vetterling, W. T. (1986). Numerical Recipes: The Art of Scientific Computing, Cambridge University Press, Cambridge.

Protter, P. E. (2004). Stochastic Integration and Differential Equations, 2nd edn, Springer-Verlag, Berlin.

Rutkowski, M. (1999). Self-Financing Strategies for Sliding, Rolling-Horizon, and Consol Bonds, Mathematical Finance 9(4), 361-385.

Schweizer, M. (1994). Risk-minimizing Hedging Strategies under Restricted Information, Mathematical Finance 4, 327-342.

Schweizer, M. (1995). On the Minimal Martingale Measure and the Föllmer-Schweizer Decomposition, Stochastic Analysis and Applications 13, 573-599.

Schweizer, M. (2001). A Guided Tour through Quadratic Hedging Approaches, in E. Jouini, J. Cvitanić and M. Musiela (eds), Option Pricing, Interest Rates and Risk Management, Cambridge University Press, 538-574. 
Sommer, D. (1997). Pricing and Hedging of Contingent Claims in Term Structure Models with Exogenous Issuing of New Bonds, European Financial Management 3(3), 269-292.

Svensson, L. E. O. (1995). Estimating Forward Interest Rates with the Extended Nelson \& Siegel Method, Penning- \& Valutapolitik 1995(3), 13-26. 